\title{
Conjurando los peligros de la metodolatría y el cientificismo en psicología
}

\begin{abstract}
Averting the perils of methodolatry and scientism in psychology
Roberto Bueno-Cuadra ${ }^{1}$

RESUMEN

Metodolatría y cientificismo son dos términos frecuentemente utilizados en algunos círculos críticos para referirse, respectivamente, a la tendencia a sobrevalorar los métodos por encima de la teoría y a la aplicación no crítica de determinados métodos de investigación. Estas dos tendencias están frecuentemente vinculadas entre sí y son una consecuencia de asumir una concepción metodológica de la ciencia. El presente artículo contribuye a alertar acerca de las limitaciones de la concepción metodológica de la ciencia y presenta un breve análisis de los niveles de la práctica científica como elaboración esquemática de los aspectos del método científico sobre los que es necesario reflexionar para evitar los riesgos que las tendencias mencionadas suponen.
\end{abstract}

PALABRAS CLAVE: Ciencia, creatividad científica, investigación, método científico, metodología.

\section{SUMMARY}

Methodolatry and scientism are two terms very used in some intellectual critical circles to refer to, respectively, a trend to overvalue methods above theory and an uncritical application of certain methods to research. These two trends are often linked between them and they are a consequence of a methodological conception of science. This article contributes to give an alert about the shortcomings of the methodological conception of science and presents a brief analysis of the levels of scientific practice as an schematic elaboration of aspects of scientific method which we have to reflect on in order to avoid the risks involved in the these trends.

KEY WORDS: Methodology, research, science, scientific creativity, scientific method.

\section{INTRODUCCIÓN}

Lo que sigue nace de la permanente preocupación del autor, como investigador y como profesor de materias que colectivamente pueden llamarse metodología de la investigación, de reflexionar acerca de los fundamentos metodológicos de la investigación científica y en particular de la investigación en psicología. Recordemos que, en realidad, la metodología no es un compendio de procedimientos, sino una fundamentación de los métodos a ser empleados en una disciplina. Son muchos los tópicos que comprende tal reflexión, pero en esta oportunidad mi atención está concentrada en dos palabras que resuenan en el campo de las ciencias sociales y la psicología de modo acusador: metodolatría y cientificismo. La primera constituye una expresión informal que alude a aquella actitud del investigador de atribuir a los métodos una garantía omnipotente para conducirlo a la verdad. Esa actitud está vinculada a una concepción metodológica de la ciencia; es decir, la idea de que hacer ciencia consiste solamente en hacer uso del "método científico", o de hacer uso de "métodos científicos" en un determinado campo de

1 Grupo de Investigación en Psicología General y Aplicada, Escuela Universitaria de Posgrado, Universidad Nacional Federico Villarreal. Lima, Perú. 
investigación. Por otro lado, von Hayek (1955) definió

el "cientificismo"

como una actitud que... involucra una aplicación mecánica y no crítica de hábitos de pensamiento a campos diferentes de aquéllos en los que fueron formados. El cientificismo... antes de considerar su objeto de estudio, reclama conocer la manera más apropiada de investigarlo. (pp. 15-16)

A mayor abundamiento, en el texto citado, von Hayek rotula como cientificismo la aceptación "mecánica y acrítica" de métodos de investigación en donde claramente, según la naturaleza del objeto de estudio, tales métodos no son los apropiados (se trata de una definición que no coincide con ninguna de las dos acepciones de esta palabra en el diccionario de la RAE, s/f). Puede haber algún vínculo entre una concepción metodológica de la ciencia y el cientificismo (al menos como lo entiende von Hayek): alguien puede creer que basta con traer a determinado campo A los métodos que se emplean exitosamente en un campo científico B para constituir A en una nueva área de estudio científico.

Mi propósito en este trabajo es, entonces, primero, pronunciarme respecto de la concepción metodológica de la ciencia. La idea fundamental a este respecto es que hacer ciencia consiste en mucho más que solamente adoptar y poner en práctica algunas reglas sobre los datos e instrumentos de investigación. En segundo lugar, y ya en relación con el tópico del cientificismo, mi segundo objetivo es distinguir los diferentes niveles de la práctica científica en los cuales el investigador puede situar su reflexión a fin de adecuar sus compromisos metodológicos a su respectivo objeto de estudio. Finalmente, me detendré brevemente a resaltar el carácter fundamentalmente inventivo de la verdadera actividad científica y, por consiguiente, el hecho de que ésta no puede limitarse únicamente a la aplicación de métodos.

\section{CIENCIA NO ES METODOLOGÍA}

Una concepción metodológica de la ciencia es la que pretende definir la ciencia por sus métodos, en vez de por sus objetivos y su contenido. En el marco de esa concepción, la investigación científica (incluida la construcción de la teoría) consiste solamente en una correcta utilización de determinadas técnicas y procedimientos que respondan en general a un determinado marco metodológico. Pero la ciencia no es fundamentalmente una cuestión de métodos, sino principalmente un intento por lograr una comprensión teórica de la realidad. Ese intento también contempla el análisis conceptual y la correcta identificación previa del objeto de estudio, más la crítica continua en torno a esos problemas. La atención casi exclusiva al componente metodológico puede derivar en un descuido completo de aspectos conceptuales importantes; como, por ejemplo, un adecuado análisis acerca de la naturaleza del propio objeto de estudio. La otra posible consecuencia de esta concepción es confundir ese marco metodológico con un verdadero análisis conceptual de la problemática a abordar. Es decir, resolver los problemas conceptuales mediante respuestas que en realidad provienen de las necesidades y requisitos exigidos por la metodología asumida. Una hermana menor de esta concepción metodológica es la concepción empirista de la ciencia, según la cual lo fundamental en ciencia son los datos, siempre que éstos hayan sido rigurosamente obtenidos.

No es desconocido que algunos de los críticos más severos de esta concepción son los autores enmarcados en una u otra visión post-modernista de la ciencia. Por ejemplo, el psicólogo construccionista Kenneth Gergen se pronunció hace tiempo en estos demoledores términos:

En un grado demasiado grande las ciencias han estado encantadas por el mito de que la aplicación asidua de un método riguroso producirá hechos sólidos -como si la metodología empírica fuera alguna forma de procesadora de carne de la cual la verdad pudiera aparecer a la manera de muchas salchichas. (1985, pp. 272-273)

En una publicación reciente, el conocido teórico subjetivista González-Rey (2020) emplea el término metodolatría para referirse a una tendencia de la psicología de mostrar mayor preocupación por los asuntos metodológicos que por los teóricos y epistemológicos. No está demás decir, sin embargo, que la denuncia de la metodolatría entre los autores post-modernistas no es por completo desinteresada. En muchos autores, esa denuncia es parte de una agenda, nada oculta, consistente en promocionar los métodos interpretativos como los únicos válidos en las ciencias sociales. La palabra misma tampoco es patrimonio de esta corriente: ya hace décadas Pepper (1942), citaba de otro autor el uso de este vocablo. En realidad, no es necesario ser un post-modernista ni ser necesariamente partidario a ultranza de los métodos interpretativos para percibir que, en efecto, no es nada saludable el énfasis casi exclusivo en los métodos y en los datos, como si esto es todo lo que contara en el desarrollo de una ciencia. 
En realidad, todos los métodos son en principio bienvenidos para mejorar nuestras observaciones acerca de los objetos y eventos, de los cuales trata, en última instancia, la ciencia. De modo que, evidentemente, el problema no puede radicar en sí en la preocupación por obtener datos de la manera más rigurosa posible. El problema es otro: "lo que debe ser cuestionado es la preocupación de tantos científicos sociales cuantitativos con los métodos, a menudo a expensas tanto de la teoría como del problema" (Berg, 2001, p. 287). En esta cita la acusación recae sobre un grupo de investigadores colectivamente identificados como "cuantitativos". Sin embargo, como argumenté en otro lugar (Bueno, 2014), el énfasis en los métodos por encima de la teoría y del tema sustantivo que se pretende investigar no es algo consustancial a las investigaciones metodológicamente enfocadas en la identificación y medición de variables (a lo cual, creo, es lo que más exactamente se puede llamar "investigación cuantitativa"). Por otro lado, es probable que lo que algunos consideran "metodolatría" es simplemente la sana y razonable escrupulosidad que se debe mantener en relación con los procedimientos cuando se hace investigación, en cuyo caso, esa palabra no es aplicable. De igual modo, y como señalan Braun y Clarke (2020), tampoco se puede acusar de "metodolatría" a los esfuerzos por sistematizar procedimentalmente un determinado enfoque de trabajo.

Pero sí hay quizá algo de razón en la sospecha de que las prácticas usuales en investigación cuantitativa pueden generar el sesgo estruendosamente denominado metodolatría. Hay dos presupuestos que pueden contribuir a generar y mantener ese sesgo. El primero de estos presupuestos es la creencia de que las prácticas usuales en investigación cuantitativa, las cuales emulan los métodos de ciencias bien reconocidas (uso de instrumentos, medición, etc.), garantizan objetividad y rigor. De ordinario, pensamos que los datos cuantitativos son más objetivos porque la información numérica es incuestionable, en el sentido de que, por ejemplo, un 25 es un 25 sin ninguna duda, a diferencia de lo que ocurriría con las apreciaciones cualitativas. Al parecer, entonces, cuando se habla de objetividad y rigor se quiere decir principalmente exactitud y precisión y en este sentido, nadie dudaría de la necesidad de que la información científica exhiba estas características. Sin embargo, ninguna metodología en particular tiene en exclusiva la facultad de producir información exacta y precisa. Dos son las cuestiones a considerar aquí: 1 . Aunque la cuantificación es indudablemente útil para representar muchos fenómenos, no es la única posible fuente de exactitud en muchos otros casos, y 2. Puede haber diferentes maneras de cuantificar en un campo determinado de estudio.

El segundo presupuesto es la idea de que en una empresa científica sólo se necesita datos "objetivos" (cuantitativos o no) obtenidos de manera rigurosa. Pero el cumplimiento de los requisitos metodológicos de exactitud y precisión, suponiendo que hayan sido definidos adecuadamente, no son suficientes en una empresa científica. Datos precisos y rigurosos no constituyen en sí mismos conocimiento. Este punto no sólo atañe a la necesidad de disponer de un "marco teórico" para realizar la "interpretación" de dichos datos, sino a algo mucho más profundo: se requiere, además, y sobre todo, de una correcta conceptualización previa del objeto de estudio y que tal conceptualización no se vea sesgada por ningún criterio metodológico asumido a priori. Los métodos no tienen validez en sí mismos ni son suficientes para justificar un área de trabajo como ciencia; por el contrario, los métodos siempre deben responder a una previa conceptualización teórica del objeto de investigación. Por tanto, no se trata simplemente de determinar qué métodos son más rigurosos y objetivos sin un previo examen conceptual de la materia. Uno de los muchos problemas que merecen consideración es, por ejemplo, el del grado en que las variables que constituyen determinadas áreas de investigación capturan la riqueza de los fenómenos psicológicos que supuestamente representan. Por supuesto, estas son advertencias que han sido lanzadas muchas veces a lo largo de décadas desde el siglo pasado. Sin embargo, es importante seguir insistiendo en ellas como recordatorio para el investigador especialmente interesado en practicar algún enfoque metodológico en particular.

No es aquí mi objetivo valorar la justeza de las diferentes metodologías hoy en boga en psicología, pero sí creo factible pronunciarme, más bien, acerca de si algún movimiento histórico en la psicología ha sido víctima de algo que pudiera llamarse metodolatría. Yo me referiré brevemente a las acusaciones frecuentes de que el conductismo ha sido fundamentalmente un movimiento metodológico dentro de la psicología, y que, además, estuvo afectado por las limitaciones inherentes a la metodología particular que lo caracterizó. Mackenzie (1977), por ejemplo, criticó la adhesión del conductismo a lo que hemos llamado el enfoque metodológico de la ciencia, explicando de ese modo su fracaso: 
Cualquier tendencia de las consideraciones metodológicas para dirigir la investigación necesita... estar subordinada a las cuestiones sustantivas particulares presentes en los casos individuales en la ciencia. No hay sustitutos metodológicos para las buenas ideas, en cada etapa de la investigación, y ninguna metodología garantizada para adquirirlas... (p. 155)

Lo que dice Mackenzie es absolutamente correcto, pero no todos los conductismos requerían ese recordatorio. La llamada de atención formulada por Mackenzie podría aplicarse esencialmente al conductismo metodológico, incluyendo aquella parte del conductismo de Watson en la que éste cuestionaba los métodos introspectivos. Pero, como insistí en otro lugar (Bueno, 1993), la cuestión fundamental del conductismo no fue nunca, ni aún en Watson, el asunto metodológico de la introspección, sino el tema de la explicación mentalista de la conducta. La dependencia de compromisos metodológicos, más que conceptuales, en el conductismo por eso así llamado metodológico, resalta fundamentalmente a la vista en su particular versión del "operacionismo": los únicos constructos válidos en psicología son los que pueden definirse operacionalmente. Skinner y Kantor fueron severos críticos de esta postura: para ellos los constructos válidos no son los que pueden definirse operacionalmente, sino los que muestran verdadera utilidad en una empresa científica. Así, en Kantor y en Skinner los preceptos metodológicos son una consecuencia de los conceptos asumidos, no al revés. De ahí que ambos rechacen el estudio de ciertos problemas, o el recurso a ciertos tipos de explicación, en este caso, los vinculados al funcionamiento de una mente, no por prurito metodológico (la falta de observabilidad o la falta de operacionalización, etc.), sino por la falta de pertinencia conceptual de dichos problemas y explicaciones.

Por otra parte, el rechazo de los conceptos mentalistas no significa dejar de lado la investigación de los fenómenos llamados mentales. Para Kantor y para Skinner, la investigación de los "procesos mentales" está al alcance de una psicología conductista. Pero la decisión de aceptar la investigación de tales eventos tampoco obedece a razones metodológicas. No hay argumento metodológico válido para excluir, por ejemplo, los eventos privados o el pensamiento o la percepción, del campo de una ciencia objetiva de la conducta (en realidad, este es el otro punto que critica Skinner, 1945, al conductismo metodológico).
Es entonces la presencia de los llamados "fenómenos mentales", no un argumento metodológico, lo que les permite formar parte del universo de fenómenos de los conductismos radical e interactivo. Sin embargo, es claro que desde la visión conductista, la investigación de esos fenómenos deberá realizarse en el marco de una conceptualización diferente de los mismos, de modo que ya no puedan entenderse como entidades internas de naturaleza no física, sino como aspectos de la propia conducta (Ribes, 1990; Ryle, 1949; Wittgenstein, 1953).

En suma, en el caso de Kantor, Skinner o Ribes la discusión metodológica no remplaza al análisis conceptual, sino que es más bien una consecuencia de éste. La concepción metodológica de la ciencia es errada, pero no puede decirse que todos los psicólogos de orientación experimental comparten esa concepción. Sus compromisos tienen un alcance conceptual mucho más profundo.

Dos comentarios finales. Primero, la crítica de la concepción metodológica de la ciencia de ninguna manera avala algún relajamiento en las exigencias metodológicas. Como queda bien claro, el objeto de tal crítica no es el rigor metodológico, sino la creencia de que éste es suficiente en cualquier área de investigación. En segundo lugar, algún lector pudiera percibir en la crítica de la concepción metodológica de la ciencia algún sentimiento desfavorable hacia la investigación empírica, y en particular hacia la cuantificación, y a la vez un temperamento más afín a la actividad filosófica. Esa sensación se incrementa cuando revisamos los trabajos de muchos autores en el campo de la psicológica teorética y filosófica (ver, por ejemplo, Teo, 2018, 2019), que es el nombre dado a la sub-disciplina formalmente abierta para la discusión de los fundamentos conceptuales de la psicología. Incluso podría verse en esta crítica una manifestación del contraste entre las dos culturas (la científica y la humanista) del cual se habló décadas atrás. Sin embargo, aunque tal impresión puede ser correcta respecto de algunos de los críticos de la concepción metodológica de la ciencia, no lo es, creo, de todos ellos. Yo puedo mencionar el caso de Emilio Ribes, psicólogo muy conocido por sus profundas reflexiones conceptuales y también crítico del empirismo ingenuo $\mathrm{y}$, sin embargo, a la vez riguroso experimentador. Ribes podría ser mencionado como un ejemplo del investigador en quien confluyen tanto el constante cuestionamiento conceptual, como la búsqueda y puesta en práctica de mejores métodos para investigar. 
Aunque quizá lo más justo sea decir que esa búsqueda está inspirada, en gran medida, precisamente, en la reflexión conceptual.

\section{LOS NIVELES DEL "MÉTODO CIENTÍFICO" COMO PRÁCTICA CIENTÍFICA}

Consideremos ahora el asunto del cientificismo. Como ya adelanté, cientificismo (al menos, como lo entiende von Hayek) y metodolatría pueden andar juntos: el traslado acrítico de métodos de un campo a otro se ve facilitado cuando en el campo receptor existe poca propensión a profundizar en los problemas conceptuales y epistemológicos implicados. Sin embargo, nuevamente, el objetivo de este trabajo no es discutir la adecuación o no de determinados métodos en algún campo particular de investigación. En esta sección en particular, me abocaré más bien a poner de relieve algunos aspectos de la práctica científica, usualmente enmarcados como "método científico", y sobre los cuales es pertinente reflexionar como parte de la búsqueda, justamente, de los métodos adecuados al campo en que el investigador se desenvuelve.

Pero, ¿qué debe entenderse por "métodos"? Una posible respuesta es hacer referencia a lo que genéricamente se ha dado en llamar método científico. El problema es que resulta bastante difícil determinar qué es el método científico, si es que lo hay. Recientemente, Cowles (2020) ha vuelto a insistir en una idea ya trasmitida por muchos otros antes que él: no existe tal cosa como el método científico y la investigación científica es algo demasiado complejo como para poder resumirse en unos cuantos pasos. Una visión más equilibrada es quizá la siguiente: "El punto aquí es que la ciencia tiene principios generales que deben ser dominados para incrementar la productividad y mejorar la perspectiva, no que estos principios proporcionan una secuencia simple y automatizada de pasos que seguir" (Gauch, 2003, p. 3). Por otro lado, también se ha cuestionado la existencia del método científico como un conjunto de principios generales, válidos en todas las disciplinas, aunque se admite que pueda hablarse de un método particular en cada una de las diferentes disciplinas especializadas. Sin embargo, incluso desde esta óptica, aún quedan dudas sobre la existencia de algún conjunto particular de normas para guiar la investigación y que aseguren "incrementar la productividad y mejorar la perspectiva". En verdad, factores como la intuición personal y el azar juegan papeles nada desdeñables en la investigación científica, como lo mostró precisamente un experimentador, como Skinner (1956), al relatar su propia experiencia como investigador.

Por lo tanto, más que referirme a una supuesta secuencia de pasos que, según se dice, no son necesariamente automatizados ni rígidos, y que incluso, pueden verse sobrepasados por factores como los mencionados por Skinner, mi objetivo en esta sección es dirigir la atención a algunos aspectos más específicos de la práctica científica. No hay método propiamente, sino una práctica guiada en parte por ciertas prescripciones, las cuales dependen, o deberían depender, de un previo análisis conceptual de la problemática a abordar, y en parte guiada por el modo propio de trabajo de cada investigador. Aun cuando la discusión que sigue es relativamente breve, confío en que será útil para al menos volver a plantearnos la confrontación de los métodos que estamos empleando en nuestros estudios con la caracterización ontológica que hayamos asumido acerca de lo psicológico.

En esta discusión no pretendo asignar al término método científico ningún significado técnico, referido a procedimientos lógicos o matemáticos particulares. Considero, más bien, que tal término ha sido objeto, más o menos informalmente, de cuatro usos distintos: 1. Como la lógica general que guía el desarrollo del conocimiento científico; 2. Como un conjunto de formulaciones o marco metodológico general de un determinado campo científico; 3. Como reglas o principios generales que garantizan la validez y la fiabilidad de los resultados de investigaciones científicas particulares y 4. Como técnicas o dispositivos específicos de obtención y análisis de datos. En cada una de estas manifestaciones el investigador debe asumir una decisión en concordancia con la naturaleza de su objeto de estudio. Puede verse, además, que estas cuatro acepciones no están al mismo nivel ya que mientras las dos primeras tienen un contenido altamente teórico (como teoría sobre los métodos), las dos últimas se refieren principalmente a aspectos más propiamente procedimentales. Como también habrá de notar el lector, los últimos niveles están comprendidos en, y dependen de, los primeros.

El término método científico, en efecto, ha sido a veces empleado para referir la lógica general de desarrollo del conocimiento científico. Desde luego, no hay acuerdo en cuanto a cuál es esta lógica. Un ejemplo muy conocido corresponde a lo que Popper (1983) llama el "método" de las conjeturas y refutaciones. En este sentido, el "método científico" 
corresponde a la formulación rigurosa de hipótesis, o conjeturas, acerca de posibles objetos o eventos todavía no conocidos, seguidas de intentos serios de refutación de tales hipótesis. Una hipótesis, en este caso, es un enunciado acerca de eventos aun no observados pero cuya existencia puede deducirse de hechos ya conocidos, o, en la ciencia más avanzada, de una teoría. Los intentos de refutación, o de crítica, de las hipótesis se llevan a cabo tanto a través de un análisis lógico de las mismas, o de las teorías de las que se deducen, como a través de su confrontación con la evidencia empírica. El objetivo no es refutar la hipótesis, sino más bien comprobar su capacidad para resistir la crítica. Una hipótesis se hace científicamente respetable, y se convierte en conocimiento cientifico (y con ella, la teoría de la que se dedujo la hipótesis), en la medida que, por el momento, y a pesar de toda la indagación más competente realizada, no se detecte en ella ningún problema lógico, ni se encuentre ninguna evidencia empírica que la contradiga. El diseño de hipótesis, por un lado, y la búsqueda de evidencia crítica, incluyendo la evidencia empírica, por el otro, no necesariamente son realizados por la misma persona. De este modo, el método científico, definido como una lógica de desarrollo de la ciencia, no comprende solamente un supuesto conjunto de reglas que un individuo concreto pone en práctica en el transcurso de un proyecto de investigación, sino que involucra una estructura de razonamiento de la actividad científica disciplinar. Otro ejemplo es el de la propuesta de Rudner (1966), para quien el método de la ciencia consiste en la búsqueda de principios generales.

El método científico también consiste en las reglas y conceptos metodológicos generales que se aceptan como válidos en un campo determinado de estudio. En psicología se pueden citar, entre muchos, cuatro ejemplos: los métodos de la psicología introspectiva de Wundt y Titchener, el método hipotético-deductivo tal como lo practicaron Clark Hull y sus asociados, los métodos operantes y la metodología de identificar y medir constructos por medio de cuestionarios. Así pues, se puede decir, por ejemplo desde la óptica de Hull, que el método científico consiste en establecer un sistema teórico a la manera de un sistema formal y en el que se identifican definiciones y postulados de los cuales se derivan teoremas que luego se someten a prueba. Tal como puede verse, en este nivel, al igual que en el anterior, nos encontramos con concepciones metodológicas que están estrechamente vinculadas con los presupuestos acerca de la naturaleza del conocimiento científico. Por ejemplo, en este nivel cabe preguntarse si el objetivo de la ciencia es siempre llegar a desarrollar un sistema hipotético-deductivo o si es posible construir un sistema hipotético deductivo que pueda capturar las propiedades esenciales, definitorias, del objeto de estudio elegido.

Como señalé, por método científico también se puede entender un conjunto de reglas o principios que aseguran en el mayor grado posible la validez y fiabilidad de la información recogida en el transcurso de una investigación. Aquí nos estamos concentrando básicamente en el problema de validar la evidencia empírica, ya sea que ésta forme parte de los argumentos en los que se basa la formulación de una hipótesis, o que se la emplee más bien para someter a crítica una hipótesis ya planteada. Desde este punto de vista, el científico tiene que resolver tres problemas fundamentales: 1.Cómo acceder de la mejor manera posible a lo que se va a observar; 2 . Cómo registrar de la manera más válida y fiable posible lo observado y 3 . Como asegurar que la evidencia empírica encontrada respalda determinada conclusión. Como se podrá notar, las decisiones metodológicas específicas a este nivel guardan correspondencia (o deberían guardarla) con las decisiones asumidas en los dos niveles previos. Por último, un cuarto sentido de método científico es el empleo de aparatos, dispositivos, instrumentos y técnicas de cálculo, así como el proceso de resumir u organizar los datos obtenidos. En este último sentido, el método científico comprende los elementos más eminentemente instrumentales y evidentemente, dichos elementos están comprendidos como partes del nivel anterior. La precaución a tener en cuenta respecto de estos dos últimos niveles del "método científico", y especialmente respecto de este último, es sencillamente que el simple uso de tales técnicas no constituye necesariamente una ciencia, punto que ampliaremos en la siguiente sección.

\section{LOS COMPONENTES DE LA PRÁCTICA CIENTÍFICA}

Nuestra crítica de la concepción metodológica de la ciencia resalta el hecho de que los métodos en sí mismos son insuficientes para hacer ciencia, dado que la primera consideración en la constitución de una investigación científica es la identificación clara y precisa de su objeto de conocimiento y el planteamiento de una teoría acerca de sus propiedades fundamentales. Pero hay otro aspecto en el que la ciencia no es únicamente aplicación de métodos. Este aspecto concierne a lo que podemos llamar el componente inventivo de la práctica científica como 
opuesto al componente instrumental o procedimental de dicha práctica.

Fue la tensión entre los componentes instrumental e inventivo la que Claude Bernard comentó tempranamente en los siguientes pasajes de su Introduction a l'etude de la médecine expérimentale: "El método experimental no dará... ideas nuevas y fecundas a los que no las tengan... el método por sí mismo no engendra nada, y es un error de ciertos filósofos el haberle otorgado demasiado poder a este respecto" (Bernard, 1865, p. 60). Páginas más abajo concluye, a partir de los enunciados anteriores, que "no podría haber un método para hacer descubrimientos" (pp. 61-62). Según estas citas, el "culto al método" ya era practicado (por algunos filósofos, dice Bernard) desde hace al menos dos siglos. Nótese que Bernard no recusa la validez del método experimental en la rama científica que él cultivaba, lo que cuestiona es la capacidad de tal método (y, agregaría yo, de cualesquier método en general), para ayudar a "generar descubrimientos", es decir, a generar ideas. Y a estas alturas bien vale recordar parte del pasaje ya citado de Mackenzie: "No hay sustitutos metodológicos para las buenas ideas... y ninguna metodología garantizada para adquirirlas.” (1977, p. 155)

En verdad, es bastante cuestionable la idea de que sea lo que sea el método científico, este pueda remplazar a las distintas habilidades intelectuales $\mathrm{y}$ actitudinales que debe poseer todo aspirante a investigador. Esas habilidades son las necesarias para dominar el componente inventivo de la investigación científica. Este corresponde a los procesos creativos, a saber: la identificación de un problema, la generación de hipótesis, el diseño de una estrategia para obtener la información que responda a la pregunta, el análisis perspicaz de los datos. Como señalaran Bloomfiel y El-Fakahani (2008):

La esencia de la investigación es la creatividad... pensamos que la investigación es acerca de empujar las fronteras más allá, abrir nuevos territorios, y pensamos sobre la creatividad como hacer algo nuevo o resolver problemas de una manera ingeniosa. Así, la investigación y la creatividad son similares, si no sinónimas. (p. 195)

Por otro lado, el componente instrumental compete al manejo de las técnicas de recolección y análisis de datos. Estos dos componentes apuntan hacia dos conjuntos muy distintos de competencias. Así, una persona puede conocer muy bien ciertas técnicas, e incluso justificar su uso hasta cierto punto sobre la base de reglas puramente metodológicas, pero esas decisiones se asumen en el contexto de objetivos y modos de trabajo que van mucho más allá de esas reglas. Una decisión a nivel inventivo se puede traducir en una decisión instrumental o procedimental, pero mientras las razones para justificar esta última son bastante claras y precisas, no siempre sucede lo mismo con las decisiones inventivas. Como dijo Murray Sidman en el prólogo de su célebre Tactics of scientific research (Sidman, 1960), el científico no siempre sabe cómo o por qué hace lo que hace.

En contra de lo que se elabora en el imaginario popular, en el que se representa al científico rodeado de instrumentos, es el componente inventivo el que está más intrínsecamente relacionado con la actividad científica. Por eso es que además de la investigación empírica existe también la investigación teórica, puramente creativa y escasamente procedimental, mientras que, en cambio, en ciencia no existe el uso de instrumentos o procedimientos en sí mismo. El uso de instrumentos se enmarca siempre en determinados objetivos que responden, a su vez (o deberían responder) a un interés teórico. Se puede saber cómo usar un microscopio o conocer un paquete estadístico, sin que ello lo convierta a uno en investigador. Los métodos, sin embargo, pueden tener un lugar en la actividad creativa cuando sirven a ésta. Incluso los propios autores cualitativistas, tan propensos a cuestionar el metodologismo, reconocen que la creatividad científica se ejerce legítimamente en relación con métodos bien fundados (May, 2006, pp. 19-20). Esto también fue resaltado por Bernard cuando declaró que, si bien el método no sirve para generar ideas, sí puede tener un impacto en un mayor o menor éxito al perseguir algún objetivo de investigación creativamente planteado (Bernard, 1865, p. 62).

De cualquier modo, es claro que mientras uno puede ser entrenado en el aspecto instrumental, no se tiene ideas claras acerca de cómo enseñar el componente inventivo. Los manuales de metodología de la investigación prestan por lo general escasa atención a este último. Existe alguna literatura en la que se proporcionan guías útiles para el ejercicio de diversos aspectos del componente inventivo (e. g., Bell, 2010; Loehle, 2010; Padilla \& Fuentes, 2017), pero yo en particular siempre tengo presente la distinción entre conducta moldeada por la contingencia y conducta gobernada por reglas (Ribes, 2000; Skinner, 1969). En gran medida, la "mente científica" todavía reclama un estudio científico. Aparte de los muchos estudios de 
caso realizados, hay relativamente poca investigación empírica, como indican Padilla y Suro (2007), acerca de cómo se adquieren y ejercen las competencias implicadas en la actividad científica, incluyendo los aspectos inventivos y también los propios procesos de razonamiento metodológico, es decir, el razonamiento para entender las reglas metodológicas (Padilla et al., 2009).

\section{CONCLUSIONES}

Mi propósito en este escrito fue aportar una contribución más a la advertencia, nunca excesiva, de asumir una actitud crítica frente a los métodos a los que estamos acostumbrados, o que pretendemos adoptar, en nuestro quehacer como investigadores en el campo del comportamiento humano. La pulcritud metodológica no puede subsanar las deficiencias en la comprensión de los fenómenos correspondientes a un área determinada, cuando esas deficiencias se deben a problemas conceptuales de origen. Es más, la confianza en los métodos puede incluso ocultar esas deficiencias a la vista del investigador. Esta reflexión es así importante para alguien que se inicia en investigación, quien muchas veces está más preocupado por el rigor de su metodología que por la claridad conceptual en su particular proyecto de investigación. Pero también lo es para la propia disciplina y los investigadores ya formados, en la medida que, a ese nivel, pareciera que no hay problemas conceptuales que resolver y se deja todo en manos de la metodología. En investigación, como en cualquier otro campo del quehacer humano, el mejor método será siempre el que esté sujeto a constante crítica.

\section{Correspondencia:}

Roberto Bueno-Cuadra.

Correo electrónico: rbueno@unfv.edu.pe

\section{REFERENCIAS BIBLIOGRAFICAS}

Bell, J. (2010). Doing your research Project. A guide for first-time researchers in education, health and social science (5ta. Ed.). McGraw-Hill/Open University Press.

Berg, B. L. (2001). Qualitative research methods for the social sciences. Allyn \& Bacon.

Bernard, C. (1865). Introduction a l'etude de la médecine expérimentale. J. B. Baillière et Fils.

Bloomfield, V. A., \& El-Fakahani, E. E. (2008). The Chicago guide to your career in science. The University of Chicago Press.
Braun, V., \& Clarke, V. (2020): One size fits all? What counts as quality practice in (reflexive) thematic analysis?. Qualitative Research in Psychology. 10.1080/14780887.2020.1769238

Bueno, R. (1993). El conductismo a los ochenta. Un análisis tridimensional. Revista Mexicana de Análisis de la Conducta, 19, 97-119. http://dx.doi. org/10.5514/rmac.v19.i1y2.23428

Bueno, R. (2014). Ciencia, ideología e investigación social: Comentarios sobre un artículo de Chavarría (2011). Actualidades en Psicología, 28(116), 6780. https://doi.org/10.15517/ap.v28i116.14893

Cowles, H. M. (2020). The scientific method. An evolution of thinking from Darwin to Dewey. Harvard University Press.

Gauch, H. G. (2003). Scientific methods in practice. Cambridge University Press.

Gergen, K. J. (1985). The social constructionist movement in modern psyhology. American Psychologist, 40(3), 266-275. https://doi. org/10.1037/0003-066X.40.3.266

González-Rey, F. (2020). Methodological and epistemological demands in advancing the study of subjectivity from a cultural-historical standpoint. Culture \& Psychology, 26(3), 562-577. https://doi. org/10.1177/1354067X19888185

Hayek, F. A. von (1955). The counter-revolution of science: Studies on the abuse of reason. The Free Press of Glencoe.

Loehle, C. (2010). Becoming a successful scientist. Strategic thinking for scientific discovery. Cambridge University Press.

Mackenzie, B. D. (1977). Behaviourism and the limits of scientific method. Routledge and Kegan Paul.

May, K. A. (2006). Conocimiento abstracto: Un caso a favor de la magia en el método. En J. M. Morse (Ed.) Asuntos críticos en los métodos de investigación cualitativa (pp.14-28). Universidad de Antioquía.

Padilla, M. A., Buenrostro, J. \& Loera, V. (2009). Análisis del entrenamiento de un nuevo científico. Universidad de Guadalajara.

Padilla, M. A., \& Fuentes, E. N. (2017). El primer paso en la formación de nuevos científicos. La elaboración de preguntas de investigación novedosas. Universidad de Guadalajara.

Padilla, M. A., \& Suro, A. L. (2007). Identificación de las competencias de investigación adquiridas por investigadores en formación. En J. J. Irigoyen, M. Jiménez y K. Acuña (Eds.), Enseñanza, aprendizaje y evaluación. Una aproximación a la pedagogía de las ciencias (pp. 137-168). Universidad de Sonora. Pepper, S. C. (1942). World hypotheses. A stydy in 
evidence. University of California Press.

Popper, K. R. (1983). Conjeturas y refutaciones. Paidós.

Real Academia Española (s/f). Cientificismo. Real Academia Española. https://dle.rae.es/ cientificismo\#3kfjE6R

Ribes, E. (1990). Psicología general. Trillas.

Ribes, E. (2000). Instructions, rules, and abstraction. A misconstrued relation. Behavior and Philosophy, 28, 41-55.

Rudner, R. (1966). Philosophy of social science. Prentice-Hall.

Ryle, G. (1949). The concept of mind. Hurchinson.

Sidman, M. (1960). Tactics of scientific research. Basic Books.

Skinner, B. F. (1945). The operational analysis of psychological terms. Psychological Review, 52(5), 270-277. https://doi.org/10.1037/h0062535
Skinner, B. F. (1956). A case history in scientific method. American Psychologist, 11, 221-233. https://doi.org/10.1037/h0047662

Skinner, B.F. (1969). Contingencies of reinforcement. A theoretical analysis. Prentice-Hall.

Teo, T. (2018). Outline of theoretical psychology. Critical investigations. Palgrave Macmillan.

Teo, T. (Ed.) (2019). Re-envisioning theoretical psychology. Diverging ideas and practices. Palgrave Macmillan.

Wittgenstein, L. (1953). Philosophical investigations. Blackwell.

Recibido: 05/10/2020

Aceptado: 30/11/2020 